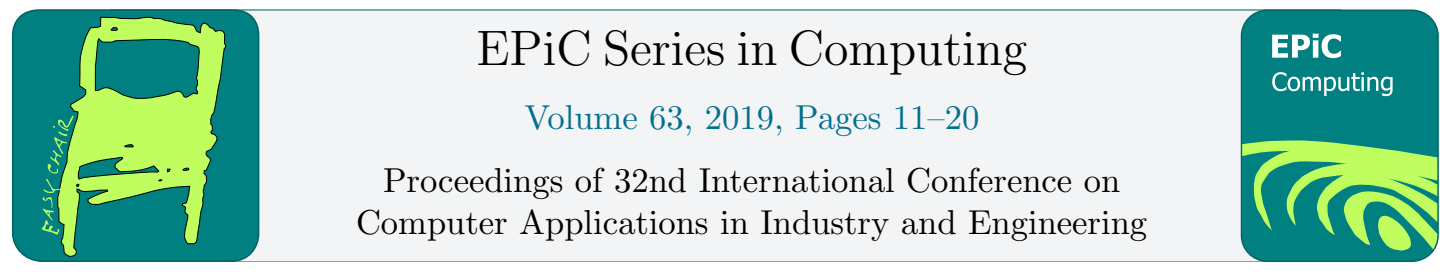

\title{
Biomedical Application of the Evolutionary Higher-Order Spectrum
}

\author{
Abdullah I. Al-Shoshan \\ Computer Engineering Dept. \\ drshoshan@gmail.com \\ Abdulmalik A. Al-Shoshan \\ Unaizah College of Medicine \\ 3bdulmalik58@gmail.com \\ Qassim University, P.O. Box 6688, Qassim 51452, Saudi Arabia
}

\begin{abstract}
Most of the biomedical signals are considered non-stationary since the human behavior depends on time. The ECG signal is one of the most important signals in cardiogram analysis. Although it provides a valuable basis for the clinical diagnosis and treatment of several diseases, it can be easily affected by various interferences caused by the power of magnetic field, patient respiratory motion or contraction. The overlapping interference affects the quality of the ECG waveform, leading to a false detection and recognition of wave groups. Therefore, the elimination of the interference of the ECG signal and the subsequent wave group identification has been a hot research topic. Since the ECG signal is not considered a stationary signal, neither the regular power spectrum nor the bispectrum can handle this problem because they do not reflect the time variation of the process characteristics. With the recent introduction of the evolutionary higherorder spectrum (EHOS) in digital signal processing, an approach for analyzing the ECG signal is proposed. The work in this paper is focusing on the reduction of the noise interferences of the ECG signal using the EHOS. This approach exploits the fact that the EHOS contains information regarding both the phase and the magnitude of the signal. Also, we will show that if the ECG signal is corrupted by stationary/non-stationary noise with symmetric distribution, the noise can be eliminated using the properties of the EHOS. Some simulation is declared to show the effectiveness of the proposed method.
\end{abstract}

\section{Introduction}

The heart, as the most important organ in the human body, is the power source of metabolism of various organs and tissues. Therefore, the extraction of high-resolution ECG signals from recordings contaminated with background noise is an important issue to investigate [1]. The electrocardiography 
(ECG) is a transthoracic interpretation of the electrical activity of the heart over time captured and externally recorded by skin electrodes. The goal of ECG signal enhancement is to separate the valid signal components from the undesired artifacts to present an ECG that facilitates easy and accurate interpretation. When there is a certain way to stimulate certain intensity through the myocardial cells, it will result in intracellular and extracellular ion flows, resulting in an action potential. ECG signals completely record this change process [2], and ECG waveform reflects the physiological conditions of various parts of the heart in medical diagnosis and is a very valuable reference for treatment [3]. The amplitude of the ECG signal is in the range of $4 \mathrm{mV}$ to $10 \mathrm{mV}$, so, it is very small and weak, and therefore, very sensitive to the effects of various disturbances [4]. One of the main sources of interference are: the loop factors, the $50 \mathrm{~Hz}$ frequency interference caused by the electromagnetic field, the baseline wander caused by the patient's limb movement, the breathing, electromyogram (EMG) interference caused by skeletal muscle stimulation, and the contraction [5]. The power frequency interference [6], the most common ECG signal interference, is caused by the magnetic field distributed by the human body from the power supply, resulting in added $50 \mathrm{~Hz}$ sinusoidal and harmonic components in the pure ECG signals [7]-[8]. With the recent introduction of the EHOS in digital signal processing [9]-[10], an approach to the analysis of a non-stationary signal problem, like an ECG signal, will be discussed when the signal is corrupted by noise. We will show that if the ECG signal is corrupted by a stationary/non-stationary noise with symmetric distribution; the noise can be eliminated using the EHOS. In the following section, we will introduce a time-frequency technique called the EHOS [9][10].

\section{The EHOS}

Since a computerized ECG signal is considered a discrete-time non-stationary signal, a time-varying spectral analysis is needed to deal with such a signal. A discrete-time non-stationary process, $x(n)$, can be represented as the output of a causal, linear, and time-varying (LTV) system, with impulse response $h(n, m)$ to a discrete-time stationary zero-mean, unit-variance white noise process [9]-[10], of the form

$x(n)=\int_{-\pi}^{\pi} H_{x}(n, \omega) e^{j \omega n} d Z(\omega)$

and $H_{x}(n, \omega)$ can be estimated as follows:

$\hat{H}(n, \omega)=\sum_{m=0}^{N-1} w_{n}(m) x(m) e^{-j \omega m}$

where

$w_{n}(m)=\sum_{i=0}^{M-1} \beta_{i}^{*}(n) \beta_{i}(m)$

and $\left\{\beta_{i}(n)\right\}$ is a set of orthonormal polynomials on $n \in[0, \mathrm{~N}-1], M$ depends on the order of nonstationarity, and $N$ is the number of samples. The EHOS is defined as follow. Let $x(n)$ be a zero-mean non-stationary process represented in equation (1). The third-order moment of $x(n)$ is given by

$R\left(n, m_{1}, m_{2}\right)=E\left\{x(n) x\left(n+m_{1}\right) x\left(n+m_{2}\right)\right\}$

which, by using equation (1), becomes 


$$
\begin{aligned}
R\left(n, m_{1}, m_{2}\right)= & \int_{-\pi}^{\pi} \int_{-\pi}^{\pi} \int_{-\pi}^{\pi} H_{x}\left(n, \omega_{1}\right) H_{x}\left(n+m_{1}, \omega_{2}\right) H_{x}\left(n+m_{2}, \omega_{3}\right) \\
& e^{j\left(\omega_{2} m_{1}+\omega_{3} m_{2}\right)} e^{j n\left(\omega_{1}+\omega_{2}+\omega_{3}\right)} E\left\{d Z\left(\omega_{1}\right) d Z\left(\omega_{2}\right) d Z\left(\omega_{3}\right)\right\}
\end{aligned}
$$

where

$E\left\{d Z\left(\omega_{1}\right) d Z\left(\omega_{2}\right) d Z\left(\omega_{3}\right)\right\}=\left\{\begin{array}{cc}S_{e}\left(\omega_{1}, \omega_{2}\right) d \omega_{1} d \omega_{2}, & \omega_{1}+\omega_{2}+\omega_{3}=0 \\ 0, & \omega_{1}+\omega_{2}+\omega_{3} \neq 0\end{array}\right.$

i.e., it vanishes except along the plane $\omega_{1}+\omega_{2}+\omega_{3}=0$, then we have that

$R\left(n, m_{1}, m_{2}\right)=\int_{-\pi}^{\pi} \int_{-\pi}^{\pi} H_{x}\left(n, \omega_{1}\right) H_{x}\left(n+m_{1}, \omega_{2}\right) H_{x}\left(n+m_{2},-\omega_{1}-\omega_{2}\right) S_{e}\left(\omega_{1}, \omega_{2}\right) e^{j\left(\omega_{2} m_{1}-\left(\omega_{1}+\omega_{2}\right) m_{2}\right)} d \omega_{1} d \omega_{2}$

and setting $m_{1}=m_{2}=0$, we get

$$
R(n, 0,0)=E\left\{x(n)^{3}\right\}=\int_{-\pi}^{\pi} \int_{-\pi}^{\pi} S_{x}\left(n, \omega_{1}, \omega_{2}\right) d \omega_{1} d \omega_{2}
$$

therefore, the EHOS of $x(n)$ is defined as

$$
S_{x}\left(n, \omega_{1}, \omega_{2}\right)=H_{x}\left(n, \omega_{1}\right) H_{x}\left(n, \omega_{2}\right) H_{x}\left(n,-\omega_{1}-\omega_{2}\right) S_{e}\left(\omega_{1}, \omega_{2}\right)
$$

Assuming that $e(n)$ is a zero-mean non-Gaussian white noise with $E\{e(n) e(n+m) e(n+k)\}=\delta(m, k)$, then $S_{e}\left(\omega_{1}, \omega_{2}\right)=1$ and therefore equation (9) becomes

$$
S_{x}\left(n, \omega_{1}, \omega_{2}\right)=H_{x}\left(n, \omega_{1}\right) H_{x}\left(n, \omega_{2}\right) H_{x}\left(n,-\omega_{1}-\omega_{2}\right)
$$

When the additive noise shown in Figure 2 is non-stationary, the traditional technique [11] will not be able to remove the noise. However, it may simply reduce the effect of non-stationary noise with symmetrically distributed like Gaussian, Laplace, uniform, and Bernoulli-Gaussian, and to remove these types of noise, we use the EHOS [9] such as

$$
S_{\text {NoisyECG }}\left(n, \omega_{1}, \omega_{2}\right)=S_{E C G}\left(n, \omega_{1}, \omega_{2}\right)+S_{\text {Noise }}\left(n, \omega_{1}, \omega_{2}\right)
$$

Therefore, in the case when the noise is non-stationary symmetrically distributed, we have [10]

$S_{\text {NoisyECG }}\left(n, \omega_{1}, \omega_{2}\right)=S_{E C G}\left(n, \omega_{1}, \omega_{2}\right)$

which means that the non-stationary noise with symmetrically distributed has no effect on the EHOS of the signal, i.e., $S_{\text {Noise }}\left(n, \omega_{1}, \omega_{2}\right)=0$.

\section{The ECG Signal}

One of the problems in biomedical data processing, like electrocardiography, is the separation of the wanted signal from noises caused by the power line interference (PLI), the external electromagnetic fields, the high-frequency interference, and the random body movements and respiration [12], [13]. In normal ECG, the time between the successive R peaks, the R-R interval, is from $0.6 \mathrm{~s}$ to $1.2 \mathrm{~s}$. The time from the onset of $\mathrm{P}$ wave and the onset of $\mathrm{R}$ wave is the P-R interval. Its duration is of $0.08 \mathrm{~s}$ to $0.12 \mathrm{~s}$. To remove signal components from unwanted frequency ranges, various types of digital filters are used [14]-[18]. It is difficult to apply filters with fixed coefficients to reduce the random noises because of the time-varying behavior of the human body. In some applications of biomedical signal processing, various useful signals are superposed by various components. The ECG signal tracking and graphical 
analysis is depicted in Figure 1 [13] and [19]. The provided image in Figure 1 (a) demonstrates the standard ECG recording paper with large boxes in heavy lines of $0.5 \mathrm{~cm}$ on the sides. On the horizontal axis, each large box represents 0.2 seconds at a typical paper speed of $25 \mathrm{~mm}$ per second, which then is divided into five smaller boxes that each represent 0.04 seconds. On the vertical axis, the large box consists of five subdivisions, each of which is $1 \mathrm{~mm}$ in height. In standard calibration, each $10 \mathrm{~mm}$ equals $1 \mathrm{mV}$. Figure 1 (c) shows a 12-lead electrocardiogram divided into the following four main contiguous lead groups: inferior, lateral, septal, and anterior. Interference caused by them may have technical sources, for example [20]:

Power line interference: This interference consists of $50 \mathrm{~Hz}$ harmonics mainly of a sinusoidal signal with an amplitude of about $50 \%$ of the peak-to-peak ECG amplitude.

Muscle contraction noise: The baseline of electromyogram is usually in the microvolt range, which makes it insignificant. Parameters are: Standard deviation is 10\% of peak-to-peak ECG amplitude; Duration is 50ms; Frequency is $10000 \mathrm{~Hz}$.
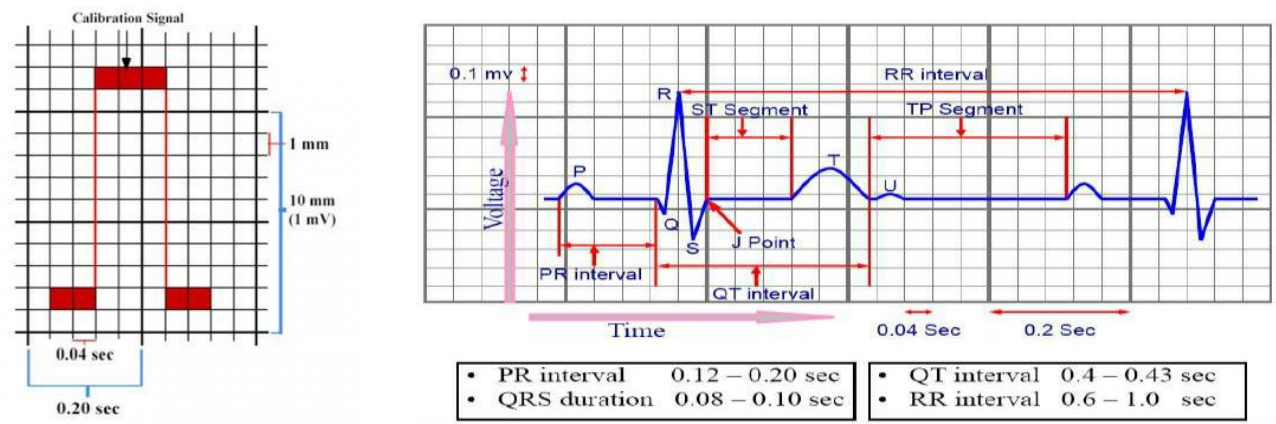

(a) Standard ECG recording paper

(b) ECG intervals

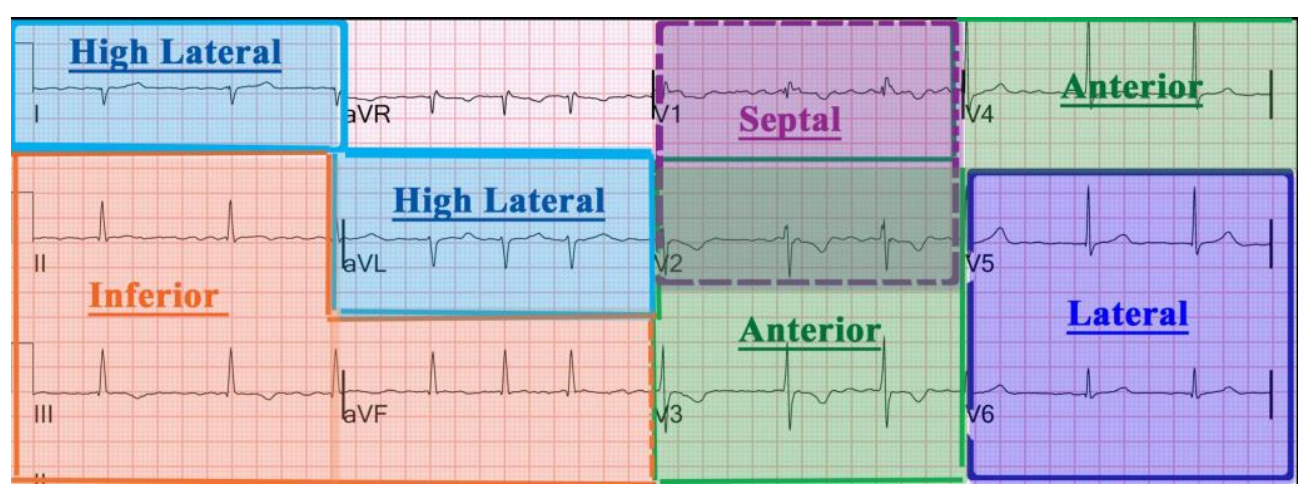

(c) A 12-lead ECG group

Figure 1: Electrocardiogram signal tracking and graphical analysis 
Electrode contact noise: This noise is caused by loss of contact between the skin and electrode, which affect the measurement of the signal. Thus, skin must be cleared and dry; also, hair -a poor conductor of electrical signal- should not prevent the contact between the electrodes and the skin by either shaving or the hair to be parted and firm contact is made [22]. The parameters are: Duration $=1 \mathrm{~s}$; Frequency = $50 \mathrm{HZ}$; Time constant is about $1 \mathrm{~s}$.

Patient movement: As ECG can also detect electrical activity of the skeletal muscle contraction, moving or shivering, or involuntary movements (e.g. those of Parkinson's disease) can mask the ECG [22]. Patient movements are transient; therefore, the baseline changes with the variations in the electrode skin impedance. Parameters are Duration between 100ms to 500ms; Amplitude is about 500\% peak-to-peak.

Electrosurgical noise: This noise destroys the ECG signal because of its large amplitude. Parameters are: Amplitude is $200 \%$ peak-to-peak; Frequency is $100 \mathrm{kHz}$ to $1 \mathrm{MHz}$; Duration is 1 to $10 \mathrm{~s}$. The ECG signal is one of the bio-signals that is considered a non-stationary signal. An efficient technique for processing and analyzing such a non-stationary signal is the time-frequency distribution.

\section{Simulation}

In this paper, some samples of a commonly used database are used. It is PhysioNet, which offers free web access to large collections of recorded physiologic signals [21]. The first example is using a normal ECG signal; however, the second example is using an abnormal ECG signal. All figures will be normalized to one for easy comparisons. Figure 2 shows a diagram for a noisy ECG signal.

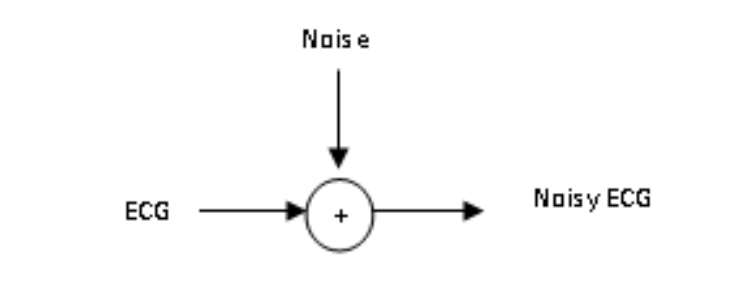

Figure 2: A noisy ECG signal

\subsection{Example 1}

Let us have the clear ECG signal of a normal ECG shown in Figure 3. In Figure 3, the P waves, QRS complexes, $\mathrm{T}$ waves, and all segments as well as intervals are normal and visible. The heart rate (HR) is 80 beats per minute (BPM) which is within the normal range and it is regular. A symmetrically distributed non-stationary random white noise with $\mathrm{SNR}=0 \mathrm{db}$ is generated and added to the clear ECG signal producing a noisy ECG signal shown in Figure 4. In Figure 3, we can clearly detect the waves, segments, as well as intervals, while, in Figure 4, the noised one, only R-R interval can be detected; however, other important clinical information are difficult to be appreciated; therefore, we aimed to remove the noise to clarify the signal. 


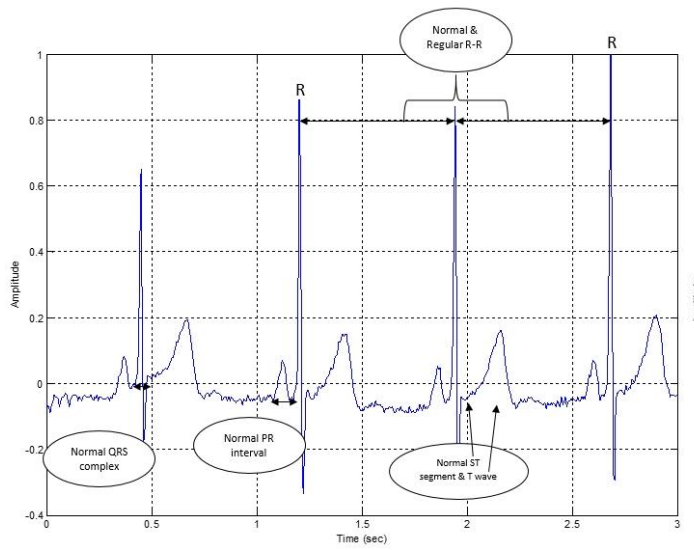

Figure 3: The original ECG signal

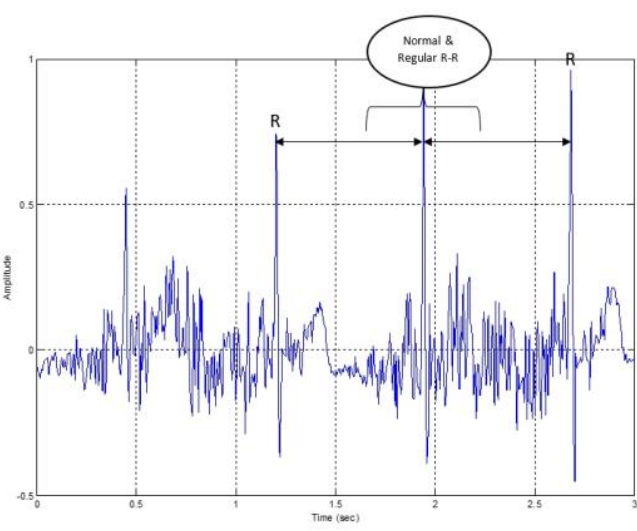

Figure 4: The noisy ECG signal

The evolutionary spectrum (ES) [9] of the noisy ECG signal is shown in Figure 5, however, one slice, at $\omega_{2}=1$, of the EHOS of the noisy ECG signal is shown in Figure 6. Comparing the two figures, we observe that the noise has been reduced or eliminated in the EHOS.

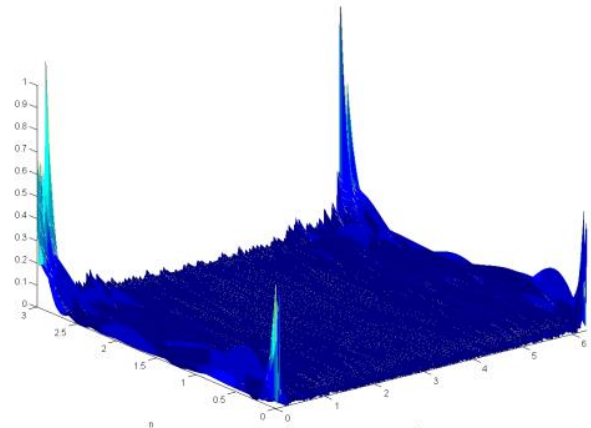

Figure 5: The ES of the noisy ECG signal

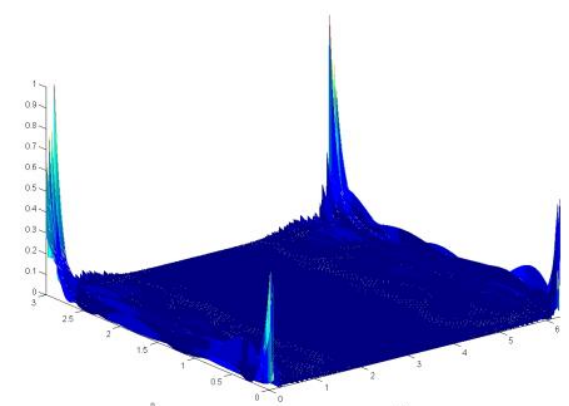

Figure 6: The EHOS of the noisy ECG signal at $w_{2}=1$

Using the EHOS to eliminate the noise, see equations (11) and (12), the estimated ECG signal is reconstructed from the EHOS using the time-dependent Fourier coefficients (TDFC) $\left\{f_{n}(u, l)\right\}$ of the EHOS estimated as follow [10]:

$$
\hat{f}_{n}(\mu, l)=\left(\frac{N}{2 \pi M}\right)^{2} \int_{-\pi}^{\pi} \int_{-\pi}^{\pi} \hat{S}\left(n, \omega_{1}, \omega_{2}\right) e^{j \omega_{1} u} e^{j \omega_{2} l} d \omega_{1} d \omega_{2}
$$

where $N$ is the length of the data sequence and $M$ is the order of the EHOS, and to get the estimated signal, equation (13) can be simplified to

$$
\hat{f}(n, 0,0)=(2 \pi)^{2} \sum_{m=0}^{N-1}\left[w_{n}(m) x(m)\right]^{3}
$$

The enhanced ECG signal is compared with the original ECG signal in Figure 7; the ECG signal before filtering is displayed in blue color and ECG signal after filtering is shown in green color; 
however, the distortion resulting from the ECG processing, which is frequently measured by the absolute error (AE), is shown in Figure 8. We observe from the figures that the noise has been reduced or eliminated.

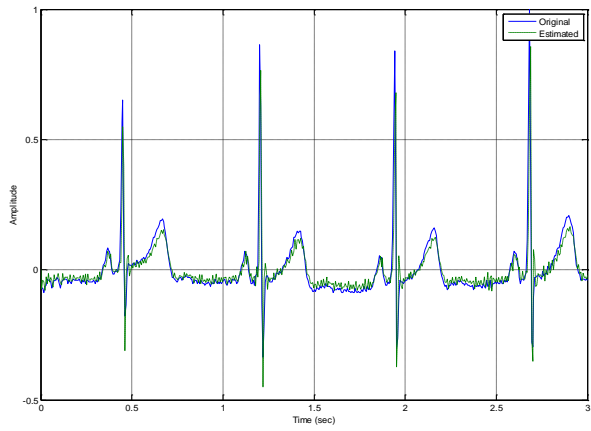

Figure 7: The enhanced vs. original ECG signals

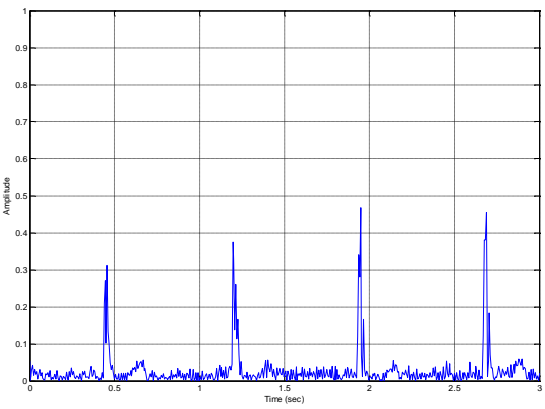

Figure 8: The AE

\subsection{Example 2}

In this example, let us have the clear signal of an abnormal ECG shown in Figure 9. Although this ECG signal has a normal and visible $\mathrm{T}$ waves with normally shaped QRS complexes, it is considered as abnormal since there are no $\mathrm{P}$ waves with irregular QRS complexes. This can happen when individual muscle fibers of the atria contract independently "fibrillating"; which is described as Atrial Fibrillation [22]. A non-stationary random white-noise with $\mathrm{SNR}=0 \mathrm{db}$ is generated and added to the original abnormal ECG signal producing the noisy ECG signal shown in Figure 10.

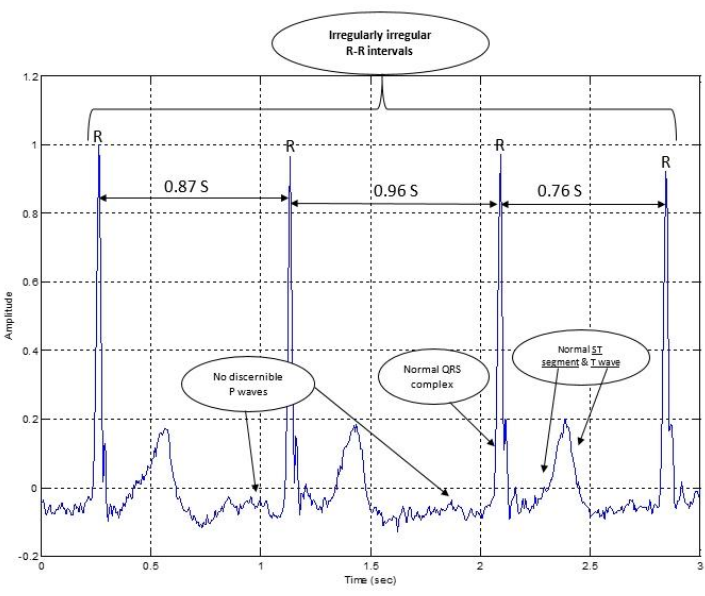

Figure 9: The original of an abnormal ECG signal

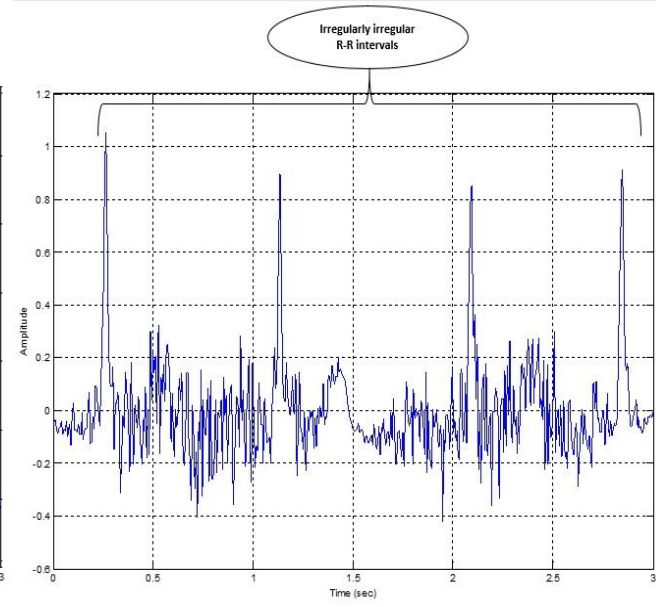

Figure 10: The abnormal noisy ECG signal

Similarly for the abnormal one shown in Figure 9, one can clearly detect the waves, segments, as well as intervals, while in the noisy one, Figure 10, only R-R interval can be detected. Other important clinical information, however, are difficult to be appreciated, which may mask the diagnosis of the abnormality (i.e. Atrial Fibrillation). Therefore, we need to eliminate or minimize the noise to clarify the signal and get a better diagnosis. 
The evolutionary spectrum (ES) of the noisy ECG signal is shown in Figure 11, however, One slice, at $\omega_{2}=1$, of the EHOS of the abnormal noisy ECG signal is shown in Figure 12. We observe from the figures that the noise has been reduced or eliminated. We should mention here that the EHOS can be applied for removing noise with symmetrical distribution only. That is because of the extensive averaging operations in calculating the EHOS. Using the EHOS to remove the noise, the estimated abnormal ECG signal is reconstructed from the EHOS using the TDFC.
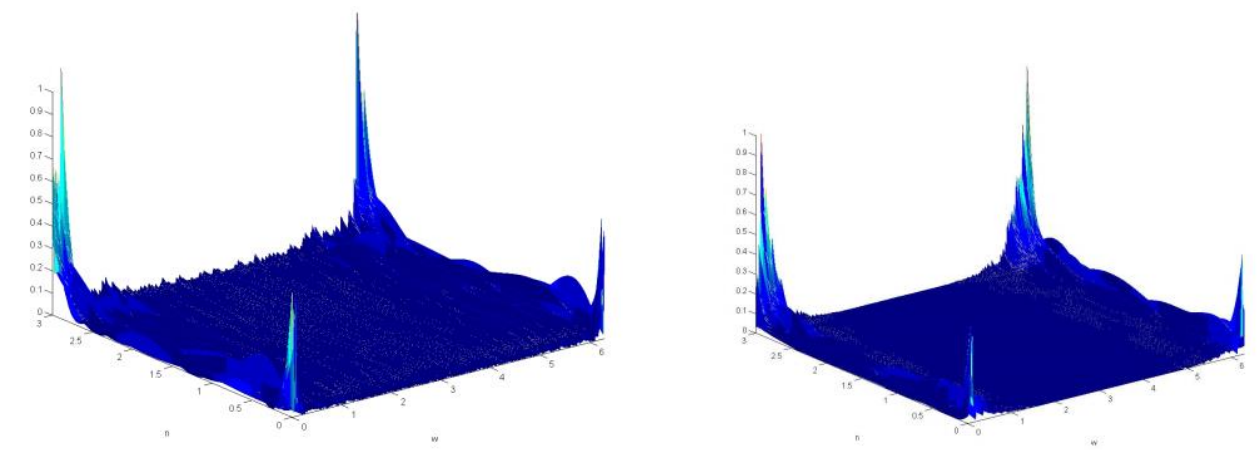

Figure 11: The ES of the noisy ECG signal

Figure 12: The EHOS of the abnormal noisy ECG at $\omega_{2}=1$

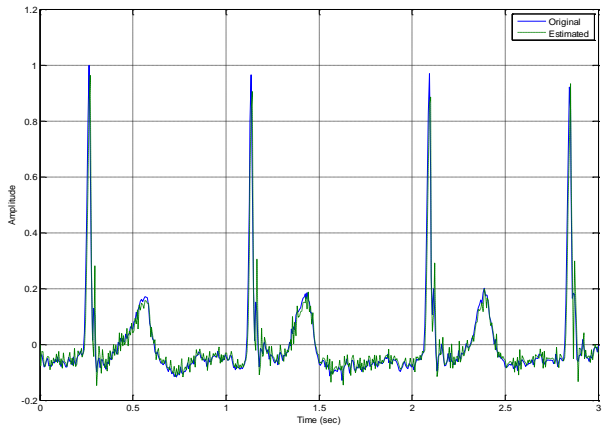

Figure 13: The enhanced vs. original abnormal ECG signals

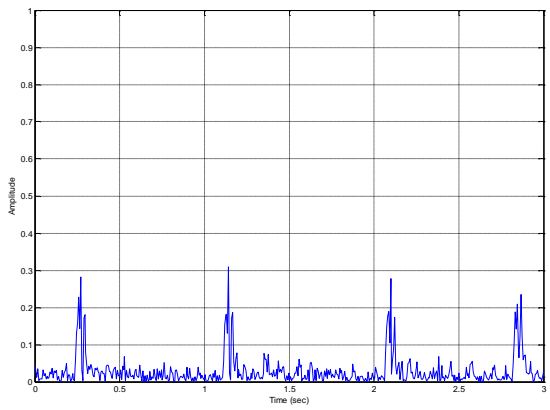

Figure 14: The AE of the abnormal ECG

The enhanced ECG signal is compared with the original ECG signal in Figure 13; the ECG signal before filtering is displayed in blue color and ECG signal after filtering is shown in green color; however, the distortion resulting from the ECG processing, which is frequently measured by the AE, is shown in Figure 14.

\section{Conclusions}

In this paper, an algorithm for processing ECG signals was introduced based on the EHOS. This approach exploits the fact that the EHOS contains information regarding both the phase and the magnitude of the signal and can be used for processing non-stationary signals. In addition, we have shown that if the ECG signal is corrupted by stationary/non-stationary noise with symmetric distribution, the noise can be removed using the EHOS. To show the effectiveness of the proposed method, some simulation was declared in two examples, one for normal ECG and another for abnormal 
ECG. The proposed approach may also be used for modeling ECG signals. The proposed algorithm can also be used for filtering more complicated biomedical signals, like electroencephalogram (EEG) signals.

\section{References}

[1] F. Sufi, F. Qiang, I. Khalil, and S. Mahmoud, "Novel methods of faster cardiovascular diagnosis in wireless telecardiology,” IEEE J. Sel. Areas Commun., vol. 27, pp. 537-552, 2009.

[2] R. Gravina, P. Alinia, H. Ghasemzadeh, and G. Fortino, "Multi-sensor fusion in body sensor networks: State-of-the-art and research challenges," Inf. Fusion, vol. 35, pp. 68-80, 2017.

[3] G. Fortino, R. Giannantonio, R. Gravina, P. Kuryloski, and R. Jafari, "Enabling Effective Programming and Flexible Management of Efficient Body Sensor Network Applications," IEEE Trans. Hum. Mach. Syst., vol. 43, pp. 115-133, 2013.

[4] Y. D. Lin and Y. H. Hu, "Power-line interference detection and suppression in ECG signal processing," IEEE Trans. Biomed. Eng. vol. 55, pp. 354-357, 2008.

[5] B. Kiremire and T. Marwala, "Nonstationarity detection: The use of the cross correlation integral in ECG, and EEG profile analysis," In Proceedings of the Congress on Image and Signal Processing (CISP'08), Sanya, China, pp. 373-378, May 2008.

[6] A. B. Sankar, D. Kumar, and K. Seethalakshmi, "Performance Study of Various Adaptive Filter Algorithms for Noise Cancellation in Respiratory Signals," Signal Process. Int. J., vol. 4, pp. $267-$ 278, 2010.

[7] R. Covello, G. Fortino, R. Gravina, A. Aguilar, and J. G. Breslin, "Novel method and real-time system for detecting the Cardiac Defense Response based on the ECG," In Proceedings of the 2013 IEEE International Symposium on Medical Measurements and Applications (MeMeA), Gatineau, QC, Canada, pp. 53-57, May 2013.

[8] J. C. Wood, A. J. Buda, and D. T. Barry. "Time-frequency transform: a new approach to first heart sound frequency dynamics," IEEE Trans. Biomedical Engineering, vol. 39(7), pp. 730-740, July 1992.

[9] M. B. Priestley, Non-linear and non-stationary time series analysis, NY: Academic Press, 1988.

[10] A. I. Al-Shoshan, "Modeling of a Non-Stationary Process Corrupted by a Symmetrically Distributed Noise Using The Evolutionary Bispectrum," In the Proceeding of the $24^{\text {th }}$ International Conference on Computer Applications in Industry and Engineering, Honolulu, Hawaii, USA, pp. 272-277, November 2011.

[11] C. L. Nikias and J. M. Mendel, "Signal processing with higher-order spectra," IEEE SP Mag., pp. 10-37, July 1993.

[12] D. K. Ojha and M. Subashini, "Analysis of Electrocardiograph (ECG) Signal for the Detection of Abnormalities Using MATLAB," International Journal of Medical, Health, Biomedical, Bioengineering and Pharmaceutical Engineering, vol. 8(2), pp. 120-123, 2014.

[13] S. Hussian and M. S. Babitha, "Noise Removal from Cardiac Signals Using Various Adaptive Algorithms," Proceedings of International Academic Conference on Electrical, Electronics and Computer Engineering, Chennai, pp. 6-9, 2013.

[14] M. Mahmud, M.S. Kaiser, A. Hussain, S. Vassanelli. "Applications of Deep Learning and Reinforcement Learning to Biological Data," IEEE Trans. Neural Netw. Learn. Syst., 2018, doi: 10.1109/TNNLS.2018.2790388.

[15] M. Huanhuan and Z. Yue, "Classification of electrocardiogram signals with deep belief networks," in Proc. IEEE $17^{\text {th }}$ International Conference on Computational Science and Engineering (CSE), pp. 7-12, Dec 2014. 
[16] Y. Yan and et al., "A restricted boltzmann machine based two-lead electrocardiography classification," in Proc. IEEE $12^{\text {th }}$ International Conference on Wearable and Implantable Body Sensor Networks (BSN), pp. 1-9, Jun 2015.

[17] Z. Wu and et al., "A novel method for classification of ECG arrhythmias using deep belief networks," International Journal of Computational Intelligence and Applications, vol. 15 (4), p. $1650021,2016$.

[18] M. Rahhal and et al., "Deep learning approach for active classification of ECG signals," Inform. Sci., vol. 345, pp. 340-354, 2016.

[19] https://emedicine.medscape.com/article/1894014-overview\#a5.

[20] R. Sehamby and B. Singh, "Noise Cancellation using Adaptive Filtering in ECG Signals: Application to Biotelemetry," International Journal of Bio-Science and Bio-Technology, vol.8(2), pp.237-244, 2016.

[21] https://physionet.org/cgi-bin/atm/ATM.

[22] J. R. Hampton, The ECG Made Easy, $8^{\text {th }}$ ed., Elsevier: Churchill Livingstone, 2013, pp. 22-27, 76-77. 